\title{
Pressure Dependence of the Fundamental Band-gap Energy of CdSe
}

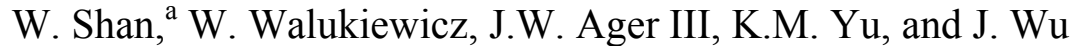 \\ Materials Sciences Division, Lawrence Berkeley National Laboratory, Berkeley, CA 94720 \\ E. E. Haller \\ Materials Sciences Division, Lawrence Berkeley National Laboratory, and Department of \\ Materials Science and Engineering, University of California, Berkeley, CA 94720
}

Pressure-dependent photo-modulation spectroscopic studies of the optical transition associated with the fundamental band gap of single-crystal bulk CdSe are presented. Photomodulated transmission (PT) measurements were performed at room temperature as a function of applied hydrostatic pressure using the diamond-anvil-cell technique. The sharp, derivative-like PT spectral features corresponding to the band-gap transition in CdSe were found to shift linearly toward higher energy with increasing pressure. By examining the pressure dependence of the PT spectra, the pressure coefficient for the direct band gap of wurtzite CdSe was determined to be $43.1 \mathrm{meV} / \mathrm{GPa}$. The hydrostatic deformation potential of the band gap was found to be $-2.3 \mathrm{eV}$.

PACS numbers: 78.30.Fs;78.55.Et;62.50. ${ }^{+} p$

a) Electronic mail: WShan@lbl.gov 
The direct band-gap semiconductor $\mathrm{CdSe}$ has been widely used to prepare nano-size crystalline clusters or quantum dots in diameters ranging from a few to a few tens of nanometers. Recently, a number of studies of the effect of applied pressure on the electronic structures and related optical properties of CdSe nanocrystals or quantum dots have been performed. ${ }^{1-9}$ In the course of these studies, the pressure dependence of the optical transitions associated with the size-dependent, quantum-confined electronic energy levels in those nanocrystals or quantum dots has been compared often with the pressure coefficients of the band gap of bulk CdSe. Such comparisons were used to estimate the pressure-induced relative changes in the total energy and the parameters associated with the change, as well as to find a better explanation for the sizedependent pressure coefficients for the optical transitions between confined electronic levels. ${ }^{8,9}$ Unfortunately, the currently available numerical values of the linear pressure coefficient in the literature varies almost by a factor of two from $37 \mathrm{meV} / \mathrm{GPa}$ derived from absorption measurement ${ }^{10}$ to $58 \mathrm{meV} / \mathrm{GPa}$ determined by photoluminescence (PL) ${ }^{11}$ Such a discrepancy will unavoidably lead to a very large uncertainty in terms of understanding the effect of pressure on the electronic structure of CdSe nanocrystals and quantum dots, especially at pressures higher than the phase transition pressure for bulk CdSe ( 3 GPa), where the band-gap energy of CdSe has to be extrapolated for the nanocrystals and quantum dots have a delayed phase transition occurring at much higher pressures. ${ }^{4,6,79}$ In addition, it has been argued recently that the larger pressure coefficient determined by PL in comparison to that by absorption may arise from the contribution of deep levels in CdSe bulk. ${ }^{8}$ For these reasons, the pressure dependence of the band-gap energy of bulk CdSe needs to be studied carefully in order to considerably reduce the existing uncertainties in regard to the pressure coefficient for the fundamental band gap of CdSe. 
In this work, we present the results of a pressure-dependent study of the optical transition associated with the fundamental band gap of CdSe bulk crystals using photomodulation spectroscopy. The sharp, derivative-like photomodulated transmission (PT) spectral features corresponding to the transition across the fundamental band gap of CdSe were found to shift linearly toward higher energy with increasing pressure, providing a direct measure of the pressure coefficients for the $\Gamma$ band-gap energy of wurtzite CdSe bulk crystals. The effective hydrostatic deformation potential of the band gap was also derived from the experimental results.

The CdSe samples used in this study are single-crystal bulk grown by the horizontal Bridgmann method. Photomodulation spectroscopy in both reflection and transmission geometries was employed to measure the band-gap energy of CdSe and its pressure dependence at room temperature $(295 \mathrm{~K})$. Quasimonochromatic light from a tungsten halogen lamp dispersed by a $0.5-\mathrm{m}$ monochromator was focused on the samples as a probe beam. A chopped $\mathrm{HeCd}$ laser beam $(4420 \AA)$ was directed onto the same spot to provide photomodulation. The spectral response signals were detected by a $\mathrm{Si}$ photodiode connected to a phase-sensitive lock-in amplification system. Application of hydrostatic pressure was accomplished by mounting small sample chips with sizes of $\sim 200 \times 200 \mu \mathrm{m}^{2}$ into gasketed diamond anvil cells. The applied pressures were determined by the standard method of monitoring the shift of the ruby R1 line.

The natural solid form of CdSe has wurtzite structure. The fundamental band gap of CdSe lies at the center of the Brillouin zone ( $\Gamma$ point). The top of the valence band is split into three spin-degenerate states $\left(\Gamma_{9}+\Gamma_{7}+\Gamma_{7}\right)$ due to crystal-field and spin-orbit coupling. Figure 1 shows two typical photomodulation spectra measured in reflection configuration with the incident light field perpendicular and parallel to the c-axis of the CdSe crystal. The derivativelike spectral lineshapes arising from the differential changes in the reflection due to 
photomodulation correspond to the optical transitions from the top of the valence bands to the bottom of the conduction band of the sample. By fitting PR spectral features to the lineshape functional form of three-dimension interband transitions, ${ }^{12,13}$ the fundamental band-gap energy $\left(\Gamma_{9 \mathrm{~V}}-\Gamma_{7 \mathrm{C}}\right.$ transition) can be determined to be $1.716 \mathrm{eV}$ for the CdSe sample at room temperature.

Shown in Fig. 2 are photomodulated transmission (PT) spectra of the CdSe sample at several selected pressures. The optical transition across the band gap shifts toward higher energy as the applied pressure increases. The PT spectral feature does not broaden significantly nor decrease in intensity drastically with increasing pressure. At pressures above $2.9 \mathrm{GPa}$, the PT spectral feature abruptly disappears as a result of the pressure-induced phase transition from wurtzite to rock-salt structure. ${ }^{2,10,11}$ The sharp, derivative-like nature of the PT spectral features allows the determination of band-gap energy under pressure with an accuracy of the order of $\mathrm{meV}$. The pressure-induced energy shifts of the optical transition related to the direct energy gap are plotted in Fig. 3. The solid line in Fig. 3 is the least-square fit to the experimental data using a linear pressure dependent fit function:

$$
E(P)=E(0)+\alpha P,
$$

where the energy $E$ is in $\mathrm{eV}$ and the pressure $P$ is in GPa. The best fit to the data yields the pressure coefficient $\alpha=43.1 \pm 1.0 \times 10^{-3} \mathrm{eV} / \mathrm{GPa}$.

Since the pressure dependence of the band gap in CdSe reported in Ref.11 was fitted to a quadratic equation:

$$
E(P)=E(0)+\alpha P+\beta P^{2},
$$

we have also performed least-square fitting to the experimental data using Eq.(2) in order to make direct comparison to results obtained by the linear fit. The dashed line in Fig. 3 is the best 
quadratic fitting result. The pressure coefficients derived from both fits to the experimental data are listed in Table I, together with the previously reported values.

The application of hydrostatic pressure, which causes a shift of the conduction-band edge relative to the valence-band edge due to the change in volume, allows a direct estimation of the effective hydrostatic deformation potential for the $\Gamma$ band gap of wurtzite CdSe. The reason for the term, effective hydrostatic deformation potential is that it is the stress, but not the strain, that is isotropic for the wurtzite structure under hydrostatic pressure. Nevertheless, based on the definition of deformation potential $\boldsymbol{a}=\partial E / \partial n V$, the variation of the band gap with pressure can be appropriately expressed in terms of the relative volume change,

$$
\Delta E=\boldsymbol{a}\left(V-V_{0}\right) / V,
$$

where $V$ is the volume, $V_{0}$ is the volume at atmospheric pressure, $\boldsymbol{a}$ is the hydrostatic deformation potential for the $\Gamma$ band gap. The relative volume change caused by applied pressure can be related by the Murnaghan equation of state: ${ }^{14}$

$$
P=\left(B / B^{\prime}\right)\left[\left(V_{0} / V\right)^{B^{\prime}}-1\right] .
$$

Where $B$ is the bulk modulus of the wurtzite CdSe and $B^{\prime}$ is its pressure derivative $(=d B / d P)$. By fitting the experimental data to Eq.(3), using recently reported values of $B=53.0 \mathrm{GPa}^{15}$ and $B^{\prime}=0.48,{ }^{15}$ the deduced effective deformation potential for the direct $\Gamma$ band gap of wurtzite CdSe was found to be $-2.30 \pm 0.03 \mathrm{eV}$. This experimental value of $\boldsymbol{a}$ is comparable to the theoretical value predicted by self-consistent local density approximation calculations $(\sim-2.1 \mathrm{eV}){ }^{15} \mathrm{It}$ should be pointed out that the value of the deformation potential deduced here from fitting experimental data depends on the numerical values of the bulk modulus and its pressure derivative. For instance, the value of $\boldsymbol{a}$ could be as small as $-1.54 \pm 0.02 \mathrm{eV}$ if the alternative values $B=35.0 \mathrm{GPa}$ and $B^{\prime}=1.1^{4}$ are used. Fortunately, we were able to make a comparison of the 
deduced deformation potential with the work reported by Gebhardt and Schötz, ${ }^{16}$ in which a hydrostatic deformation potential of $-2.7 \pm 0.2 \mathrm{eV}$ for zinc-blende CdSe epilayer was reported. Thus, we regard the result of $\boldsymbol{a} \approx-2.30 \pm 0.03 \mathrm{eV}$ as consistent and more accurate estimate.

In conclusion, we have studied the effect of hydrostatic pressure on the fundamental band gap of wurtizte CdSe bulk using photomodulation spectroscopy in conjunction with diamondanvil pressure-cell technique. By monitoring the shift of the sharp, derivative PT spectral feature that corresponds to the transition from the topmost valence band to the bottom of the conduction band with pressure, we are able to determine the pressure coefficient $\mathrm{d} E / \mathrm{d} P=43.1 \pm 1.0$ $\mathrm{meV} / \mathrm{GPa}$ for the direct band gap of wurtzite CdSe and derive the numerical value of $\boldsymbol{a} \approx-$ $2.30 \pm 0.03 \mathrm{eV}$ from the experimental results for the effective hydrostatic deformation potential associated with the band gap.

This work is supported by the Director, Office of Science, Office of Basic Energy Sciences, Division of Materials Sciences and Engineering, of the U.S. Department of Energy under Contract No. DE-AC03-76SF00098. 


\section{References}

1. A.P. Alivisatos, T.D. Harris, L.E. Brus, and A. Jayaraman, J. Chem. Phys. 89, 5979(1988).

2. S.H. Tolbert, A.P. Alivisatos, J. Chem. Phys. 102, 4642(1994).

3. S.H. Tolbert, A.B. Herhold, C.S. Johnson, and A.P. Alivisatos, Phys. Rev. Lett. 73, 3266(1994).

4. S.H. Tolbert, A.B. Herhold, L.E. Brus, and A.P. Alivisatos, Phys. Rev. Lett. 76, 4384(1996).

5. J. Schroeder and P.D. Persans, J. Luminescence, 70, 69(1996).

6. B.S. Kim, M.A. Islam, L.E. Brus, and I.P. Herman, J. Appl. Phys. 89, 8127(2001).

7. J.B. Li, G.H. Li, J.B. Xia, J.B. Zhang, Y. Lin, and X.R. Xiao, J. Phys.: Condens. Matter, 13, 2033(2001).

8. R.W. Meulenberg and G.F. Strouse, Phys. Rev. B66, 035317(2002).

9. W. Shan, W. Walukiewicz, K.M. Yu, J. Wu, J.W. Ager III, and D. Ning, unpublished.

10. A.L. Edwards and H.G. Drickamer, Phys. Rev. 122, 1149(1961).

11. J.R. Mei and V. Lemos, Solid State Comm. 52, 785(1984).

12. D. E. Aspnes, in Optical Properties of Solid, edited by M. Balkanski (North-Holland, Amsterdam, 1980), Chap. 4A.

13. O. J. Glembocki, SPIE Proc. Vol.1286, 2(1990).

14. F.D. Murnaghan, Proc. Natl. Acad. Sci. 30, 244(1944).

15. S.H. Wei and A. Zunger, Phys. Rev. B60, 5404(1999).

16. W. Gebhardt and G. Schötz, in Properties of Wide Bandgap II-VI Semiconductors (EMIS Datareview Series No.17), ed. R. Bhargava, p.113(INSPEC, London, 1995). 
Table I. Pressure coefficients for the fundamental band gap of wurtzite CdSe bulk

\begin{tabular}{cccc}
\hline \hline Experimental Method & $E(0)(\mathrm{eV})$ & $\begin{array}{c}\alpha=d E / d P(\mathrm{eV} / \mathrm{PGa}) \\
\left(\times 10^{-3}\right)\end{array}$ & $\begin{array}{c}\beta=d^{2} E / d P^{2}\left(\mathrm{eV} / \mathrm{PGa}^{2}\right) \\
\left(\times 10^{-3}\right)\end{array}$ \\
\hline PT (linear fit) $^{\mathrm{a}}$ & $1.715 \pm 0.001$ & $43.1 \pm 1.0$ & - \\
PT (quadratic fit) & $1.713 \pm 0.002$ & $48.1 \pm 3.1$ & $-1.9 \pm 1.1$ \\
Absorption $^{\mathrm{b}}$ & - & 37 & - \\
PL $^{\mathrm{c}}$ & 1.738 & 58 & -5 \\
\hline \hline
\end{tabular}
a. This work
b. Reference 10
c. Reference 11 


\section{Figure Captions}

Fig. 1. Photoreflectance spectra of wurtzite CdSe bulk at room temperature.

Fig. 2. Photomodulated transmission spectra recorded at selected pressures.

Fig. 3. Change of the band-gap energy of CdSe with pressure. The solid line is the best linear fit to the data. The dashed line represents the result of the best quadratic fitting. 


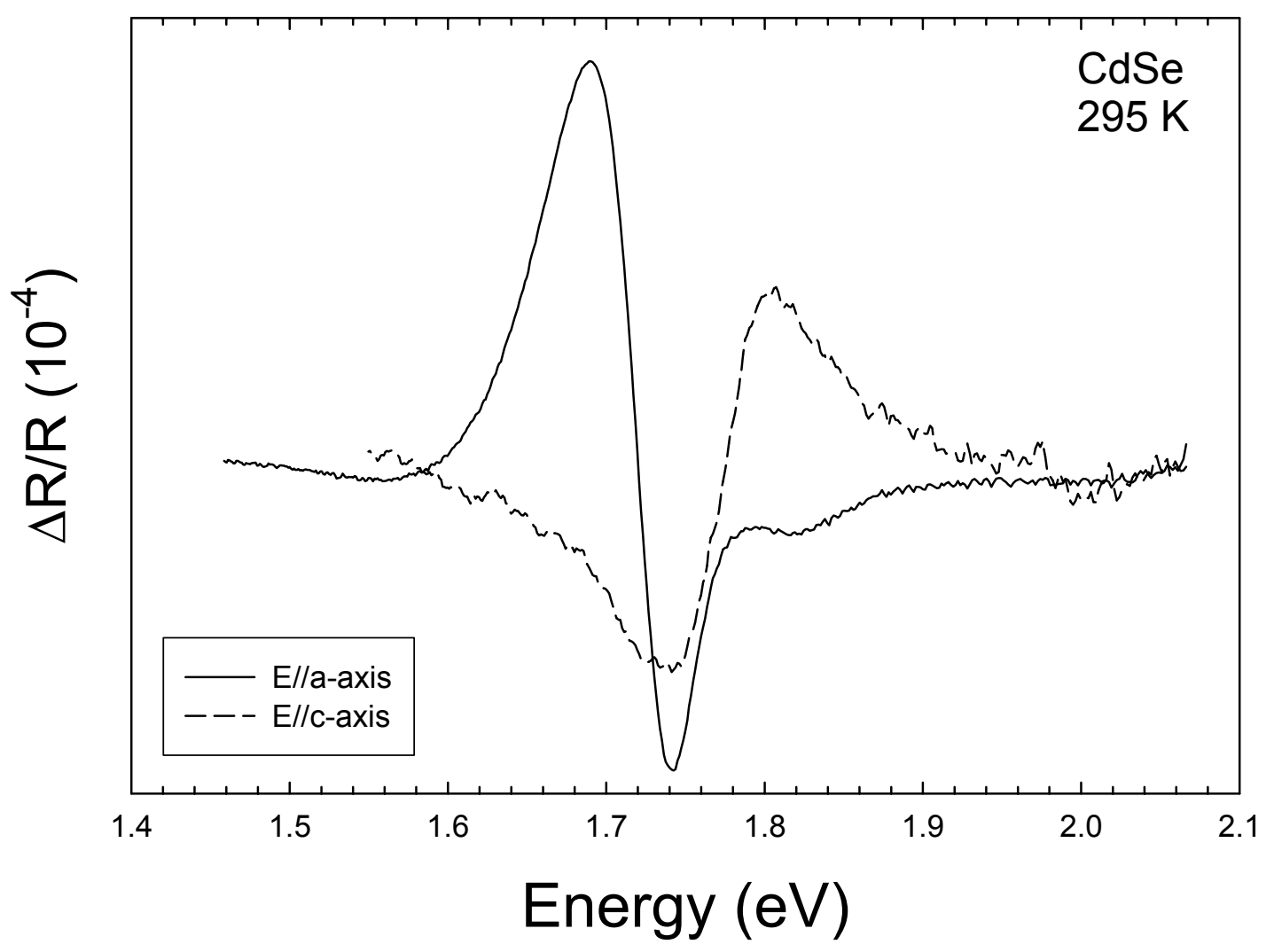

Fig. 1/3 


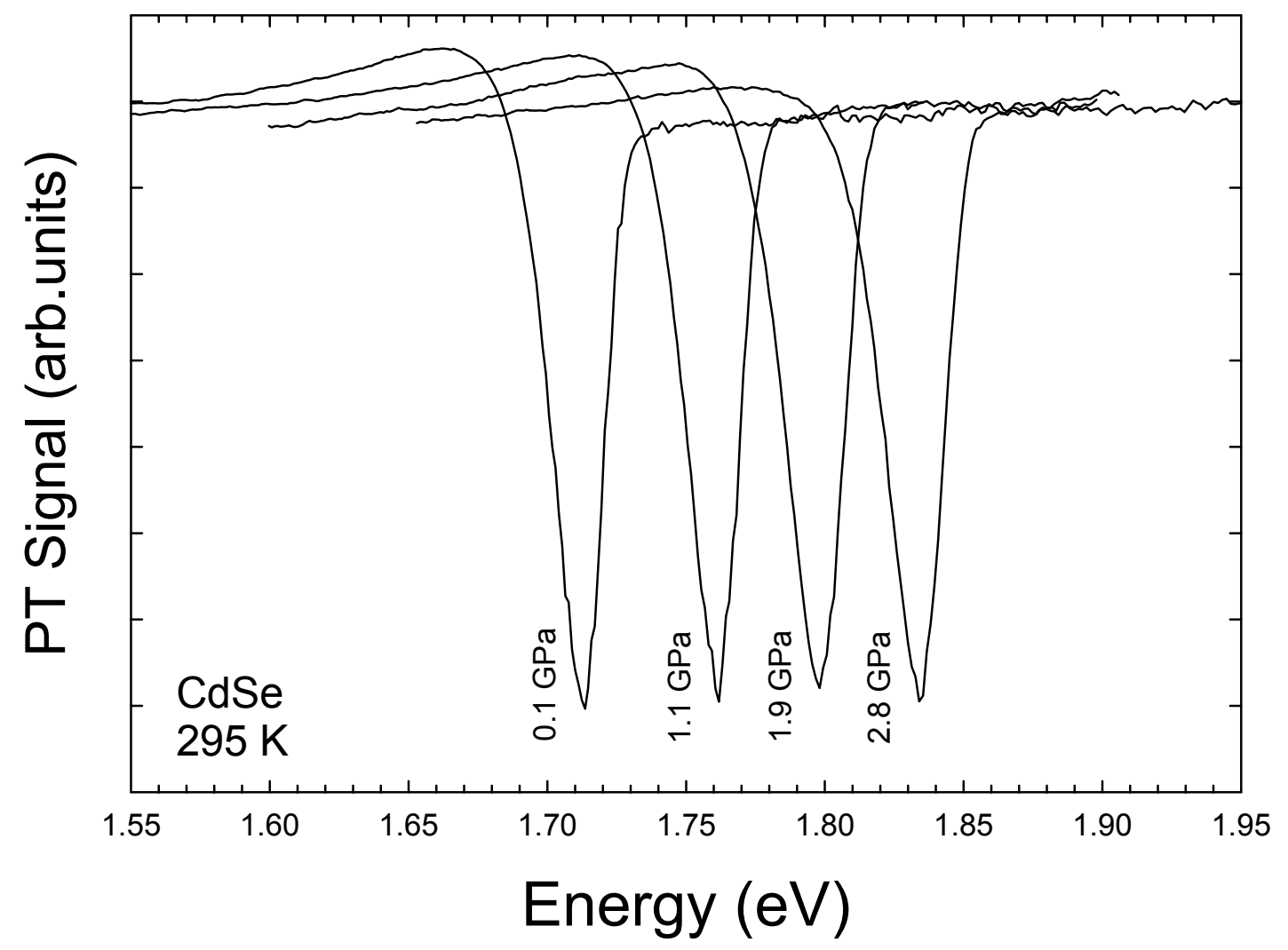

Fig. 2/3 


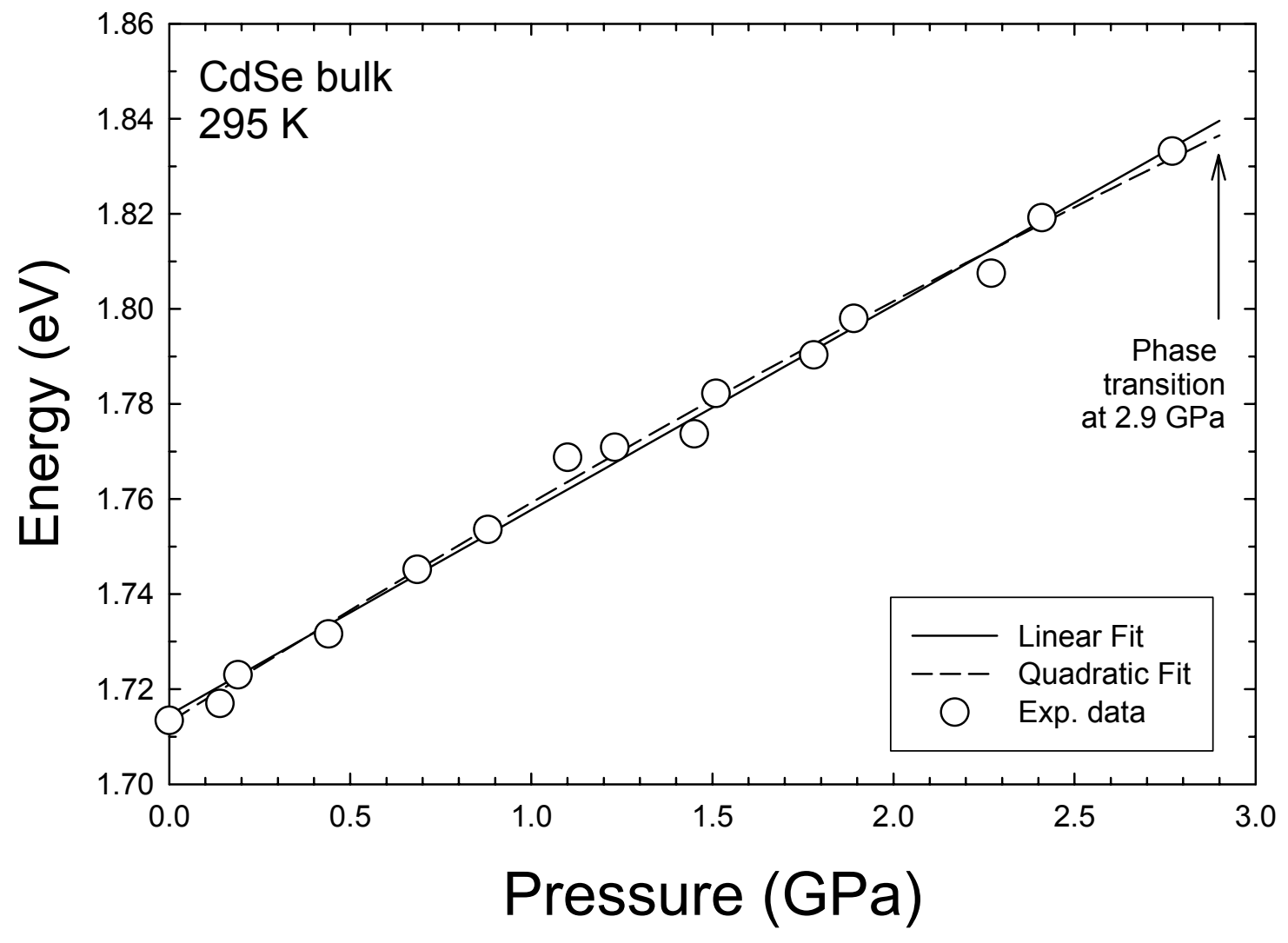

Fig.3/3 